\title{
Iterative Frequency-Domain Channel Estimation and Equalization for Ultra-Wideband Systems with Short Cyclic Prefix
}

\author{
Salim Bahçeci and Mutlu Koca (EURASIP Member) \\ Wireless Communications Laboratory, Department of Electrical and Electronics Engineering, Boğaziçi University, \\ Bebek, 34342 Istanbul, Turkey \\ Correspondence should be addressed to Mutlu Koca, mutlu.koca@boun.edu.tr
}

Received 4 October 2009; Revised 5 March 2010; Accepted 9 June 2010

Academic Editor: Cihan Tepedelenlioğlu

Copyright ( $\odot 2010$ S. Bahçeci and M. Koca. This is an open access article distributed under the Creative Commons Attribution License, which permits unrestricted use, distribution, and reproduction in any medium, provided the original work is properly cited.

\begin{abstract}
In impulse radio ultra-wideband (IR-UWB) systems where the channel lengths are on the order of a few hundred taps, conventional use of frequency-domain (FD) processing for channel estimation and equalization may not be feasible because the need to add a cyclic prefix (CP) to each block causes a significant reduction in the spectral efficiency. On the other hand, using no or short $\mathrm{CP}$ causes the interblock interference (IBI) and thus degradation in the receiver performance. Therefore, in order to utilize FD receiver processing UWB systems without a significant loss in the spectral efficiency and the performance, IBI cancellation mechanisms are needed in both the channel estimation and equalization operations. For this reason, in this paper, we consider the joint FD channel estimation and equalization for IR-UWB systems with short cyclic prefix (CP) and propose a novel iterative receiver employing soft IBI estimation and cancellation within both its FD channel estimator and FD equalizer components. We show by simulation results that the proposed FD receiver attains performances close to that of the full CP case in both line-of-sight (LOS) and non-line-of-sight (NLOS) UWB channels after only a few iterations.
\end{abstract}

\section{Introduction}

Recently, frequency-domain (FD) processing for receiver design has gained considerable interest, particularly in single-carrier (SC) communication systems because of the significant complexity reductions it offers while attaining the same as and often better performances than those of the time-domain (TD) methods $[1,2]$. Ordinarily, to be able to employ FD processing at the receiver, a cyclic prefix (CP) that is at least as long as the channel is added to each transmitted data block such that the linear convolution of the channel and the transmitted data block can be expressed as an equivalent circular convolution operation and an FD signal model can be derived. In the FD signal model, the channel distortion appears as a single tap fading coefficient and the FD channel estimation and equalization algorithms can be implemented with simple arithmetic operations in contrast to the complex matrix inversions required by their time-domain counterparts [3].

Because of these memory/computational complexity reductions, FD processing has also emerged as powerful design tool for impulse radio ultra-wideband communication (IR- UWB) systems, which are characterized by long delay spreads [4]. For instance, minimum mean-squared error (MMSE) frequency-domain equalization (FDE) is proposed for IR-UWB and direct sequence- (DS-) UWB transmissions and their performances are compared in [5]. An SC IR-UWB system employing FD equalization (FDE) is proposed in [6], as an alternative to the multiband OFDM UWB appoaches. The proposed method achieves lower peakto-average power ratios than that of the MB-OFDM UWB systems and is more effective in collecting multipath energy and combatting the intersymbol interference (ISI). In [7, 8], zero-forcing and MMSE FD detectors are proposed for IR-UWB systems and compared with the classical RAKE receiver. An iterative FDE for IR-UWB systems is proposed in [9] based on energy spreading transform. Finally, an FD turbo equalization and multiuser detection scheme is presented in [10] for the DS-UWB systems. Notice that these works present the FDE methods for UWB with the underlying assumption that channel impulse response (CIR) for the multipath UWB channel is available whereas the joint 
FD UWB channel estimation and equalization problem is addressed in [11] for SC-FDE UWB and in [12] for DS-UWB systems.

In all the works mentioned above, full CP (that is at least as long as the channel) is assumed to be inserted between transmitted blocks. However, for UWB channels where the delay spread is very large, adding full CP means a significant degradation in the spectral efficiency and throughput. On the other hand, using short or no CP for spectral efficiency causes a mismatch between the linear and circular convolution operations and thus the interblock-interference (IBI) between the transmitted blocks. Therefore the IBI reconstruction and cancellation must be incorporated in the FD receiver design so that low complexity FD algorithms can be used without the need for full CP, which has been addressed in the related context, that is, for SC communications in [13-17] and for UWB communications in [18-20]. In [13], a reduced-CP SC-FDE system is proposed where the CP length is reduced using specifically designed frame structures. Iterative reconstruction of the missing $\mathrm{CP}$ is proposed and its performance on the FDE is evaluated in [14] again for SC communications. In [15], FD channel estimation problem is addressed in the presence of insufficient CP and an interference cancellation and channel estimation algorithm is proposed for SC block transmission and this method is applied to turbo equalization in [16]. Similarly, a joint iterative FD channel estimation and equalization scheme is presented for SC-FDE without CP in [17]. Regarding the UWB literatures, a CP reconstruction and FDE algorithm for IR-UWB communication is proposed with known channel coefficients in [18] based on the CP reconstruction method presented in [21] and the impact of imperfect channel estimation is presented in [19]. A different approach is also proposed in [20], where a time-division multiple access scheme is incorporated with the SC-FDE over UWB channels so as to cancel the multiple access interference and IBI effects that is due to insufficient CP, again assuming the channel knowledge is available.

To place the related works in the literature into perspective, please notice that in order for frequency-domain processing to be feasible for UWB communications, the receiver design problem needs to be addressed in a uniform framework encompassing the following criteria: (1) frequency-domain processing for joint channel estimation and equalization for low complexity, (2) reduced or no CP to avoid significant loss in spectral efficiency, and (3) IBI suppression to retrieve the performance loss due to the lack of full CP (possibly via iterative processing). Unfortunately, most of the works mentioned above address one or more of these design issues, but not all of them. For this reason, we present in this paper a novel FD iterative UWB receiver architecture that preserves the spectral efficiency of UWB systems while recovering possible performance losses due to IBI with very low complexity. The low complexity is partially also due to the fact that even though the receiver is iterative, the performance gains are attained after only a few iterations.

The proposed iterative receiver consists of three softinput soft-output (SISO) blocks: a channel estimator implemented by the FD recursive least squares (RLSs) algorithm, a minimum mean squared error (MMSE) FDE, and a repetition decoder to extract soft bit values from the pulse repetitions. The channel estimator makes an estimate of the IBI using subsequent pilot blocks in each recursion that is removed from the received signal model before a recursion of the channel estimation update is made. At the end of the pilot mode, the channel estimate is passed onto the back-end iterative receiver that is comprised of the SISO MMSE equalizer and the repetition decoder. The SISO MMSE equalizer performs soft cancellation of both IBI and ISI at its input and soft log-likelihood mapping at its output. The joint equalization and decoding iterations are carried out so as to improve the soft decisions on transmitted bits. Notice that contrary to the conventional approach, the SISO repetition decoder within the iterative receiver is not a module to decode an outer code but instead an inherent part of the UWB symbol detection architecture. The proposed iterative receiver is simulated for both lineof-sight (LOS) and nonline-of-sight (NLOS) UWB channels and simulation results indicate that even with very short $\mathrm{CP}$ lengths, it achieves performances that are very close to those of the full CP cases by using relatively small number of pilot blocks. Moreover, simulations also indicate that the proposed receiver performs significantly well even when there is no $\mathrm{CP}$ used at the transmitter side.

The rest of this paper is organized as follows: The FD signal model is presented in Section 2. Then the proposed iterative receiver structure with the IBI estimation and cancellation is presented in Section 3. Section 4 is devoted to the simulation results, and the paper is ends in Section 5 with some conclusive remarks.

\section{Signal Model}

We consider a single user uncoded chip-interleaved directsequence pulse amplitude modulated UWB (DS-PAMUWB) system [22], where every symbol is transmitted over a duration of $T_{s}$ with $N_{f}$ frames each with a duration of $T_{f}$, that is, $T_{s}=N_{f} T_{f}$. As indicated in [22], in a DS-PAM UWB system the chip duration is equal to the frame duration $\left(T_{f}=\right.$ $\left.T_{c}\right)$; that is, every frame has one chip $\left(N_{f}=N_{c}\right)$. In each chip, a pulse $p(t)$ with a duration of $T_{p}=T_{c}$ is transmitted. The $n$th input information sequence having $N_{b}$ bits is represented as $b_{n}=\left\{b_{n}(l)\right\}_{l=0}^{N_{b}-1}$ where $b_{n}(l) \in\{+1,-1\}$. Each bit is spread to $\mathbf{d}_{n}^{\prime}=\left\{d_{n}^{\prime}(k)\right\}_{k=0}^{N-1}$ by repeating every bit $N_{c}$ times where $N=N_{b} N_{c}$. Thus $d_{n}^{\prime}(k)$ can be expressed as

$$
\mathbf{d}_{n}^{\prime}(k)=b_{n}\left(\left\lfloor\frac{k}{N_{c}}\right\rfloor\right), \quad k=0, \ldots, N-1,
$$

where $\lfloor\cdot\rfloor$ denotes the integer floor operation. Then $\left\{d_{n}^{\prime}(k)\right\}_{k=0}^{N-1}$ is interleaved to $\mathbf{d}_{n}=\left\{d_{n}(k)\right\}_{k=0}^{N-1}$. For FD processing at the receiver, the last $L_{p}$ elements of $\mathbf{d}_{n}$ are inserted to the beginning of the sequence as the CP. Then the transmitted signal is expressed as

$$
s_{n}(t)=\sum_{k=-L_{p}}^{N-1} g_{n}(k) p\left(t-k T_{c}\right)
$$


where $g_{n}(k)=d_{n}\left(\langle N+k\rangle_{N}\right)$, and $\langle\cdot\rangle_{N}$ is the modulo operation with respect to $N$.

The multipath channel is modelled as

$$
h(t)=\sum_{i=0}^{\widetilde{L}-1} \rho_{i} \delta\left(t-\tau_{i}\right),
$$

where $\tilde{L}$ is the number of channel paths, and $\rho_{i}$ and $\tau_{i}$ are the path gain and the delay of the $i$ th path, respectively. The path delays $\tau_{i}$ can be approximated as integer multiples of $T_{c}$ for simplicity and the CIR can be written as

$$
h(t)=\sum_{l=0}^{L_{c}-1} h(l) \delta\left(t-l T_{c}\right),
$$

where $L_{c}=\tau_{\tilde{L}-1} / T_{c}+1$ with $\tau_{\widetilde{L}-1}$ being the maximum path delay, and $h(l)=\rho_{i}$ for $l=\tau_{i} / T_{c}$ and zero for all other $l$ values. Assuming that the receiver is fully synchronized and time delays are known, the received signal for $n$th block can be expressed as

$$
r_{n}(t)=s_{n}(t) * h(t)+w_{n}(t)
$$

where $*$ denotes linear convolution and $w_{n}(t)$ is AWGN with variance $N_{0} / 2$. The received signal is passed through a chip-matched filter and sampled at the chip rate $T_{c}$. After the removal of $\mathrm{CP}$ the discrete time received signal can be expressed as

$$
r_{n}(k)=s_{n}(k) \star h(k)+w_{n}(k), \quad k=0, \ldots N-1,
$$

where $r_{n}(k), s_{n}(k), h(k)$, and $w_{n}(k)$ are the samples of chipmatched filter output, transmitted signal, discrete-time CIR, discrete-time noise sample, respectively, and $\star$ represents circular convolution. If the $\mathrm{CP}$ length is shorter than the CIR $L_{p}<L_{c}$, an IBI error term is added to the received signal such that

$$
r_{n}(k)=s_{n}(k) \star h(k)+e_{n}(k)+w_{n}(k), \quad k=0, \ldots N-1,
$$

where

$$
\begin{aligned}
e_{n}(k) & \\
\quad= & \sum_{r=L_{p}+k+1}^{L_{c}-1} h(r)\left[d_{n-1}\left(N+L_{p}+k-r\right)-d_{n}(N-r+k)\right]
\end{aligned}
$$

for the first $L_{c}-L_{p}-1$ terms (i.e., for $k=0,1, \ldots L_{c}-L_{p}-2$ ) and 0 for the rest. The derivation of the IBI term in (8) is given in the Appendix. The signal model in (7) can be written in block form as

$$
\mathbf{r}_{n}=\mathbf{D}_{n} \mathbf{h}+\mathbf{e}_{n}+\mathbf{w}_{n},
$$

where $\mathbf{h}$ is an $(N \times 1)$ channel coefficient vector that is zero padded after the first $L_{c}$ terms, and $\mathbf{r}_{n}, \mathbf{e}_{n}$, and $\mathbf{w}_{n}$ are $(N \times 1)$ column vectors collecting samples obtained from the received signal, the IBI error terms, and the samples obtained from the AWGN, respectively, such that

$$
\begin{aligned}
& \mathbf{h}=\left[\begin{array}{lllllll}
h(0) & h(1) & \cdots & h\left(L_{c}-1\right) & 0 & \cdots & 0
\end{array}\right]^{T}, \\
& \mathbf{r}_{n}=\left[\begin{array}{lllll}
r_{n}(0) & r_{n}(1) & r_{n}(2) & \cdots & r_{n}(N-1)
\end{array}\right]^{T}, \\
& \mathbf{e}_{n}=\left[\begin{array}{lllll}
e_{n}(0) & e_{n}(1) & e_{n}(2) & \cdots & e_{n}(N-1)
\end{array}\right]^{T}, \\
& \mathbf{w}_{n}=\left[\begin{array}{lllll}
w_{n}(0) & w_{n}(1) & w_{n}(2) & \cdots & w_{n}(N-1)
\end{array}\right]^{T} .
\end{aligned}
$$

$\mathbf{D}_{n}$ is a circular matrix whose first column is expressed as

$$
\mathbf{d}_{n}=\left[\begin{array}{lllll}
d_{n}(0) & d_{n}(1) & d_{n}(2) & \cdots & d_{n}(N-1)
\end{array}\right]^{T},
$$

and the other columns are obtained by circularly shifting first column downwards. Since $\mathbf{D}_{n}$ is a circular matrix, it can be written as

$$
\mathbf{D}_{n}=\mathbf{F}^{H} \mathbf{C}_{n} \mathbf{F},
$$

where $\mathbf{F}$ is an $(N \times N)$ discrete Fourier transform (DFT) matrix and $\mathbf{C}_{n}$ is an $(N \times N)$ diagonal matrix whose $m$ th diagonal entry is

$$
\begin{aligned}
C_{n}(m) \\
=\sum_{k=0}^{N-1} d_{n}(k) \exp \left(-j \frac{2 \pi}{N} m k\right) \quad m=0, \ldots N-1 .
\end{aligned}
$$

After the DFT the $n$th received signal blocks can be expressed as

$$
\mathbf{R}_{n}=\mathbf{C}_{n} \mathbf{H}+\mathbf{E}_{n}+\mathbf{W}_{n},
$$

where $\mathbf{H}=\mathbf{F h}, \mathbf{E}_{n}=\mathbf{F e}_{n}$ and $\mathbf{W}_{n}=\mathbf{F w}_{n}$.

\section{Iterative FD Channel Estimation and Equalization with IBI Cancellation}

The block diagram of the proposed iterative receiver is shown in Figure 1. The channel estimator makes an initial estimation of the channel coefficients in the presence of IBI due to insufficient CP. Prior to each subsequent recursion, the IBI is estimated and removed from the received signal in (9), and the resulting signal is employed in the channel estimation step. At the end of the pilot-aided channel estimation stage, the estimated channel coefficients are passed onto the back-end iterative receiver that consists of the SISO MMSE equalizer and the SISO repetition decoder. Notice that in the initial equalization iteration, the information symbols are not available and the equalization is performed without the IBI cancellation. However following the initial pass, the SISO MMSE equalizer computes a soft estimate of the IBI that is to be used in the soft IBI and ISI cancellation prior to the equalization. In the following both the front-end FD channel estimator block and the back-end iterative channel equalizer/decoder are presented in detail. 


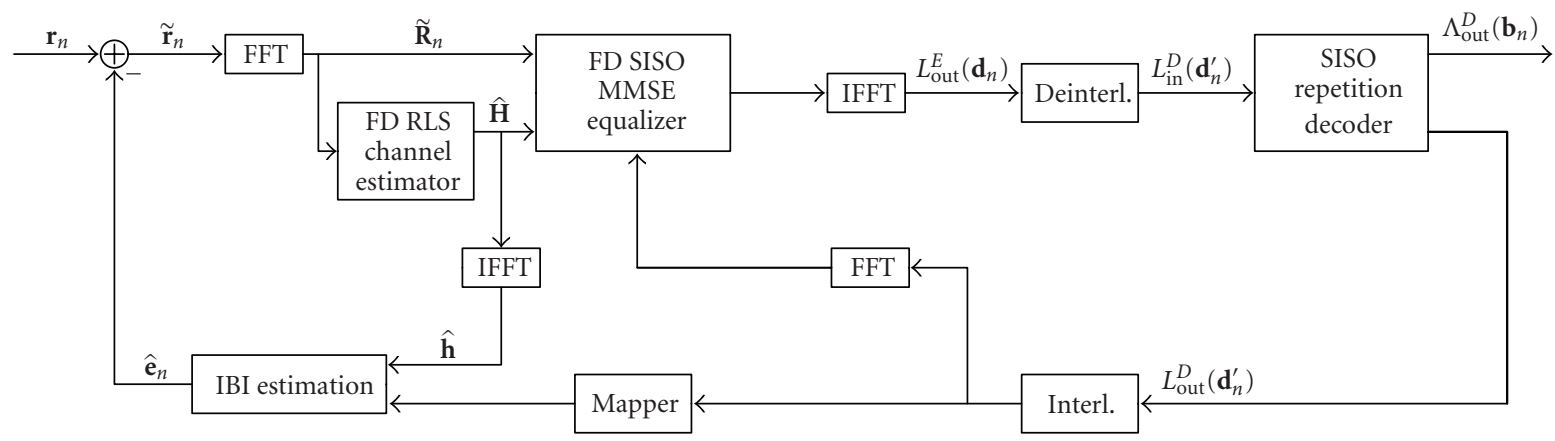

FIGURE 1: Block diagram of the proposed receiver.

3.1. FD Channel Estimation. The FD channel estimator with IBI cancellation appears at the front-end of the receiver block diagram in Figure 1. In the proposed receiver, FDRLS algorithm described in [23] is employed for its fast convergence and for smaller pilot overhead. However, a less complex channel estimator can also be used such as the FD LMS algorithm without changing the receiver architecture. Given the model in (14), the FD-RLS channel estimator aims to minimize the cost function:

$$
J_{\mathrm{RLS}}(H)=\sum_{i=1}^{n} \lambda^{n-i}\left\|\mathbf{R}_{n}-\mathbf{C}_{n} \mathbf{H}\right\|^{2}, \quad n=1, \ldots, N_{p},
$$

where $0<\lambda<1$ is the forgetting factor and $N_{p}$ is the number of pilot blocks. The minimum is achieved for $\hat{\mathbf{H}}_{n}=\mathbf{H}$, with $\hat{\mathbf{H}}_{n}$ satisfying the recursive equation:

$$
\hat{\mathbf{H}}_{n}=\hat{\mathbf{H}}_{n-1}+\mathbf{K}_{n} \mathbf{z}_{n}, \quad n=1, \ldots N_{p} .
$$

Here, $\mathbf{z}_{n}=\mathbf{C}_{n}^{H}\left[\mathbf{R}_{n}-\mathbf{C}_{n} \hat{\mathbf{H}}_{n-1}\right]$ and $\mathbf{K}_{n}=\operatorname{diag}\left[K_{n}(0) K_{n}(1)\right.$ . $\left.K_{n}(N-1)\right]$ where

$$
\begin{gathered}
K_{n}(m)=\frac{S_{n-1}(m)}{\lambda+\left|C_{n}(m)\right|^{2} S_{n-1}(m)}, \\
n=1, \ldots, N_{p}, \quad m=0, \ldots, N-1,
\end{gathered}
$$

with $S_{n}(m)$ computed by the recursive relation:

$$
\begin{array}{r}
S_{n}(m)=\frac{1}{\lambda} S_{n-1}(m)\left[1-K_{n}(m)\left|C_{n}(m)\right|^{2}\right], \\
n=1, \ldots N_{p}, \quad m=0, \ldots, N-1 .
\end{array}
$$

The first pilot block is used to make an initial estimate of the channel without any IBI cancellation. Once this estimate $\widehat{\mathbf{h}}$ is available, it is used to compute an estimate of the nonzero IBI terms:

$$
\begin{aligned}
\hat{e}_{n}(k) & \\
\quad= & \sum_{r=L_{p}+k+1}^{L_{c}-1} \hat{h}(r)\left[d_{n-1}\left(N+L_{p}+k-r\right)-d_{n}(N-r+k)\right] .
\end{aligned}
$$

Notice that the IBI term in (19) differs from the one in (8) in employing the channel estimates instead of the real values.

After the transmission of the first pilot block, the estimated IBI is cancelled from the received signal to yield the new TD signal representation

$$
\widetilde{\mathbf{r}}_{n}=\mathbf{D}_{n} \mathbf{h}+\mathbf{e}_{n}-\widehat{\mathbf{e}}_{n}+\mathbf{w}_{n},
$$

or equivalently in the FD

$$
\widetilde{\mathbf{R}}_{n}=\mathbf{C}_{n} \mathbf{H}+\mathbf{E}_{n}-\widehat{\mathbf{E}}_{n}+\mathbf{W}_{n} .
$$

Then, subsequent recursions of the FD-RLS algorithm are carried out by replacing $\mathbf{R}_{n}$ in (15) by $\widetilde{\mathbf{R}}_{n}$ of (21) and cancelling the IBI estimation successively.

In the decision-directed mode where soft-estimates on the data symbols are available, the nonzero terms of the IBI error are estimated using these soft-values as

$$
\begin{array}{r}
\hat{e}_{n}^{i}(k) \\
\quad=\sum_{r=L_{p}+k+1}^{L_{c}-1} \hat{h}(r)\left[\bar{d}_{n-1}\left(N+L_{p}+k-r\right)-\bar{d}_{n}(N-r+k)\right],
\end{array}
$$

where $\bar{d}_{n-1}$ and $\bar{d}_{n}$ denote the soft bit values corresponding to the $(n-1)$ st and $n$th data blocks, respectively. Notice that these soft values are computed from the log likelihood ratios (LLRs) via the hyperbolic tangent function $\tanh (\cdot)$, that is, $\bar{d}_{\mathrm{n}}(k)=\tanh \left(L\left(d_{n}(k)\right) / 2\right)$ as presented in detail in the sequel.

As for the complexity of the channel estimation algorithm, the FFT and IFFT operations for a sequence of length $N$ requires approximately $2 N \log _{2} N$ real multiplications and $2 N \log _{2} N$ real additions. As seen from Figure 1, in each channel estimation recursion one FFT and one IFFT is required. Another FFT operation is required for the transformation of the time-domain IBI term into frequency-domain. Notice that exact computation of the $\tanh (\cdot)$ function can be costly, however it can be done via the piecewise linear approximations or coarse quantization approaches with look up table [24]. Using the piecewise linear approximation in [24] with 8 regions, the computation of the soft symbols costs roughly about 2 real additions and 1 multiplication per symbol. Considering also the subtraction inside the 
parenthesis in (19) and the multiplication outside, the computation of the IBI term and the cancellation operations requires $3 N$ real products and $4 N$ real additions. Finally one recursion of the channel estimation algorithms employs $22 \mathrm{~N}$ products and $15 \mathrm{~N}$ additions for FD-RLS and $14 \mathrm{~N}$ products and $13 N$ additions for FD-LMS [23]. As a result, the overall computational complexity of the FD-RLS channel estimator is $6 N \log _{2} N+25 N$ real multiplications and $6 N \log _{2} N+19 N$ real additions per pilot block. The complexity of FD-LMS channel estimator would be slightly lower as it requires $6 N \log _{2} N+17 N$ real multiplications and $6 N \log _{2} N+17 N$ real additions, however its convergence is significantly slower. For this reason the FD-RLS algorithm is employed for channel estimation throughout the simulations.

3.2. Iterative FD Equalization and Decoding. The back-end iterative receiver is comprised of a FD-SISO-MMSE equalizer [25], SISO repetition decoder similar to proposed in [26] and an IBI estimation block. The estimated CIR coefficients, received information and the extrinsic a priori LLR of each chip position obtained by interleaving the LLRs of the decoder, $L_{\text {in }}^{E}\left(d_{n}(k)\right)=\prod\left(L_{\text {out }}^{D}\left(d_{n}^{\prime}(k)\right)\right)=\ln \mathbf{P}\left\{d_{n}(k)=\right.$ $1\} / \mathbf{P}\left\{d_{n}(k)=-1\right\}$ are fed to the FD SISO MMSE equalizer. The estimate of each chip position is computed as $\bar{d}_{n}(k)=$ $\tanh \left(\left(L_{\text {in }}^{E}\left(d_{n}(k)\right)\right) / 2\right)$ where as mentioned before $\tanh (\cdot)$ denotes the hyperbolic tangent function. Then the decision at the output of the FD equalizer is

$$
\begin{aligned}
\hat{D}_{n}(m)= & \frac{\hat{H}(m) \hat{H}(m)^{*}}{\sigma_{w}^{2}+\hat{H}(m) \hat{H}(m)^{*}} \widetilde{R}_{n}(m) \\
& +\left(\mu-\frac{\hat{H}(m) \hat{H}(m)^{*}}{\sigma_{w}^{2}+\hat{H}(m) \hat{H}(m)^{*}}\right) \bar{D}_{n}(m), \\
& \quad m=0,1, \ldots N-1,
\end{aligned}
$$

where $\sigma_{w}^{2}$ is the signal-to-noise ratio (SNR), $\hat{H}(m)$ is the $m$ th frequency component of the estimated channel coefficient, $\bar{D}_{n}(m)$ is the $m$ th frequency component of estimated chip position, and $\mu$ is defined as

$$
\mu=\frac{1}{N} \sum_{m=0}^{N-1} \frac{\hat{H}(m) \hat{H}(m)^{*}}{\sigma_{w}^{2}+\hat{H}(m) \hat{H}(m)^{*}} .
$$

The equalizer produces LLRs $L_{\text {out }}^{E}\left(d_{n}(k)\right)$ of each chip position as

$$
L_{\text {out }}^{E}\left(d_{n}(k)\right)=\frac{2 \hat{d}_{n}(k) \mu}{\hat{\sigma}^{2}},
$$

where $\hat{d}_{n}(k)$ is the estimated value of $k$ th chip position in time-domain, and $\hat{\sigma}^{2}$ is expressed as

$$
\hat{\sigma}^{2}=\frac{1}{N} \sum_{k=0}^{N-1}\left|\operatorname{sign}\left(\hat{d}_{n}(k)\right) \cdot \mu-\hat{d}_{n}(k)\right|^{2} .
$$

The obtained LLRs are deinterleaved and fed to the SISO repetition decoder as inputs $L_{\text {in }}^{D}\left(d^{\prime}{ }_{n}(k)\right)=\prod-1\left(L_{\text {out }}^{E}\left(d_{n}(k)\right)\right)$.
In the decoder the a posteriori LLR output for $\ell$ th bit of the $n$th block $b_{n}(\ell)$ is computed as

$$
\Lambda_{\text {out }}^{D}\left(b_{n}(\ell)\right)=\sum_{j \in \chi_{\ell}} L_{\text {in }}^{D}\left(d_{n}^{\prime}(j)\right),
$$

where $\chi_{\ell}=\left\{N_{c} \ell, N_{c} \ell+1, \ldots, N_{c} \ell+N_{c}-1\right\}$ containing chip positions related to the $\ell$ th bit. The extrinsic LLR for the chip $d_{n}^{\prime}(j)$ associated with $b_{n}(\ell)$ is given by

$$
L_{\text {out }}^{D}\left(d_{n}^{\prime}(j)\right)=\Lambda_{\text {out }}^{D}\left(b_{n}(\ell)\right)-L_{\text {out }}^{D}\left(d_{n}^{\prime}(j)\right) .
$$

After interleaving, this extrinsic information is sent to the SISO FD equalizer and IBI estimator. The IBI estimation is done by using expected values of each chip positions and they are calculated as

$$
\bar{d}_{n}(k)=\tanh \left(\frac{L_{\mathrm{out}}^{D}\left(d_{n}(k)\right)}{2}\right) .
$$

In order to cancel the IBI error term, an approach similar to that proposed for channel estimation can be used. The IBI error can be estimated and subtracted from the received symbol in the next iteration as shown in Figure 1. However, for symbol detection the transmitted symbols are not known; so the IBI error estimation cannot be done as in (19). For this case, the expected values of the previously transmitted symbols which are obtained as in (29) can be used to estimate the nonzero terms of the IBI error as

$$
\begin{aligned}
& \hat{e}_{n}^{i}(k) \\
& \quad=\sum_{r=L_{p}+k+1}^{L_{c}-1} \hat{h}(r)\left[\bar{d}_{n-1}^{I}\left(N+L_{p}-1+k-r\right)-\bar{d}_{n}^{i}(N-r+k)\right]
\end{aligned}
$$

for $k=0,1, \ldots, L_{p}-L_{c}-2$ where $i$ and $I$ are the iteration index and the total number of iterations per each received block, respectively.

Considering again the computational complexity for the back-end iterative equalizer, the IFFT and FFT operations require $4 \mathrm{Nlog}_{2} \mathrm{~N}$ real multiplications and $4 \mathrm{Nlog}_{2} \mathrm{~N}$ real additions in each iteration. The cost of FD MMSE equalization with IBI estimation and cancellation is $10 \mathrm{~N}$ real products and $4 \mathrm{~N}$ real additions per iteration. In simulations, convergence of the iterative receiver is observed after only two iterations, meaning that the increase in complexity due to the number of iterations is low. The complexity brought by the interleaving/deinterleaving operations and by the repetition decoder is much lower than the equalizer and channel estimator blocks, and thus it is neglected in this discussion.

\section{Simulation Results}

In this section simulation results of the proposed receiver structure are presented with different CP lengths over the UWB channel models CM1-CM4 proposed in [4] where CM1 is a line-of-sight (LOS) channel whereas CM4 is a nonline-of-sight (NLOS) channel with a long delay spread. 


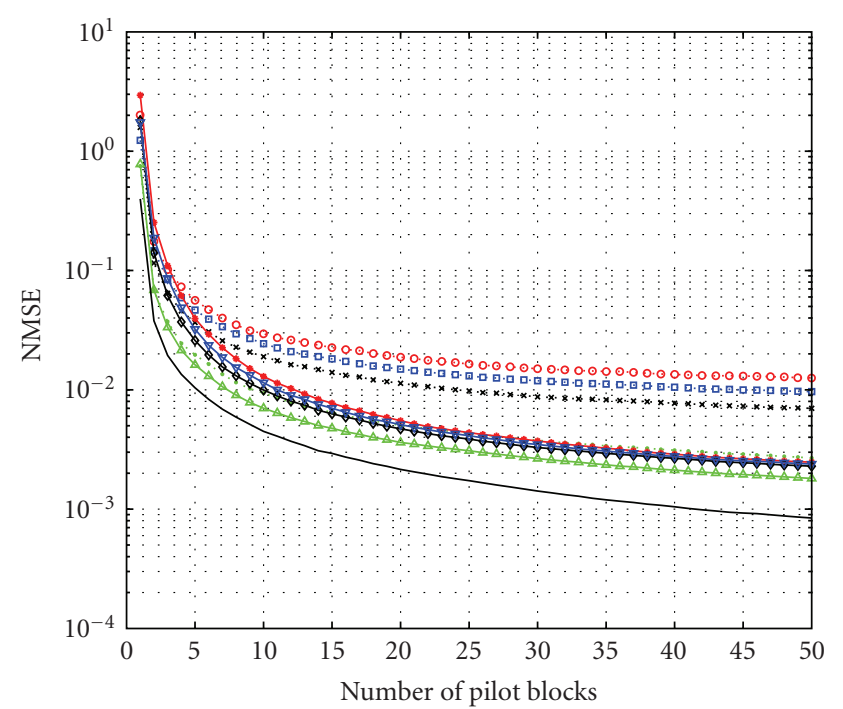

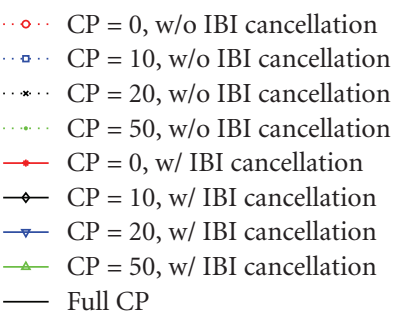

FIgURE 2: Performance of FD channel estimation with and without IBI cancellation, number of pulse repetition $=4$ block size $=160$ symbols (640 chips), max. CM4 UWB channel, channel length $=360$, and taps $\mathrm{SNR}=20 \mathrm{~dB}$.

All channel models are simulated via computer trials and run over 1000 channel realizations. Both the pulse and chip durations are chosen as 1 nanosecond, that is, $T_{p}=$ $T_{c}=1$ nanosecond. In each block 160 information bits are transmitted where each bit is spread over 4 chips. At the receiver side, matched filter outputs are sampled at the chip-rate, so each received block has 640 samples. In each channel realization, the channel impulse response changes in each run. However, for the full cyclic prefix conditions, the maximum channel spreads are assumed to be $110,160,200$, and 360 taps for the CM1-CM4 channels, respectively.

The performance of the channel estimator is measured over the CM4 channel model by the normalized mean squared error (NMSE) at its output that is defined as $\operatorname{NMSE}(\hat{\mathbf{H}}) \triangleq \mathrm{E}\left\{\|\mathbf{H}-\hat{\mathbf{H}}\|^{2}\right\} / \mathrm{E}\left\{\|\mathbf{H}\|^{2}\right\}$. Figure 2 shows the NMSE of the channel estimator performances with or without the IBI cancellation for $\mathrm{CP}$ lengths of $0,10,20$, and 50 and full $\mathrm{CP}$ conditions at $20 \mathrm{~dB}$ SNR. Notice from the figure that, the use of short $\mathrm{CP}$ or no $\mathrm{CP}$ degrades the performance of the FD-RLS algorithm significantly. However, the IBI cancellation algorithm employed with the channel estimator partially compensates this performance loss. Without the IBI cancellation, employing a $\mathrm{CP}$ of 50 symbols improves the channel estimation performance almost half an order of magnitude compared to the zero $\mathrm{CP}$ case, which is far more than that of the length 10 or 20 symbol CP. However,
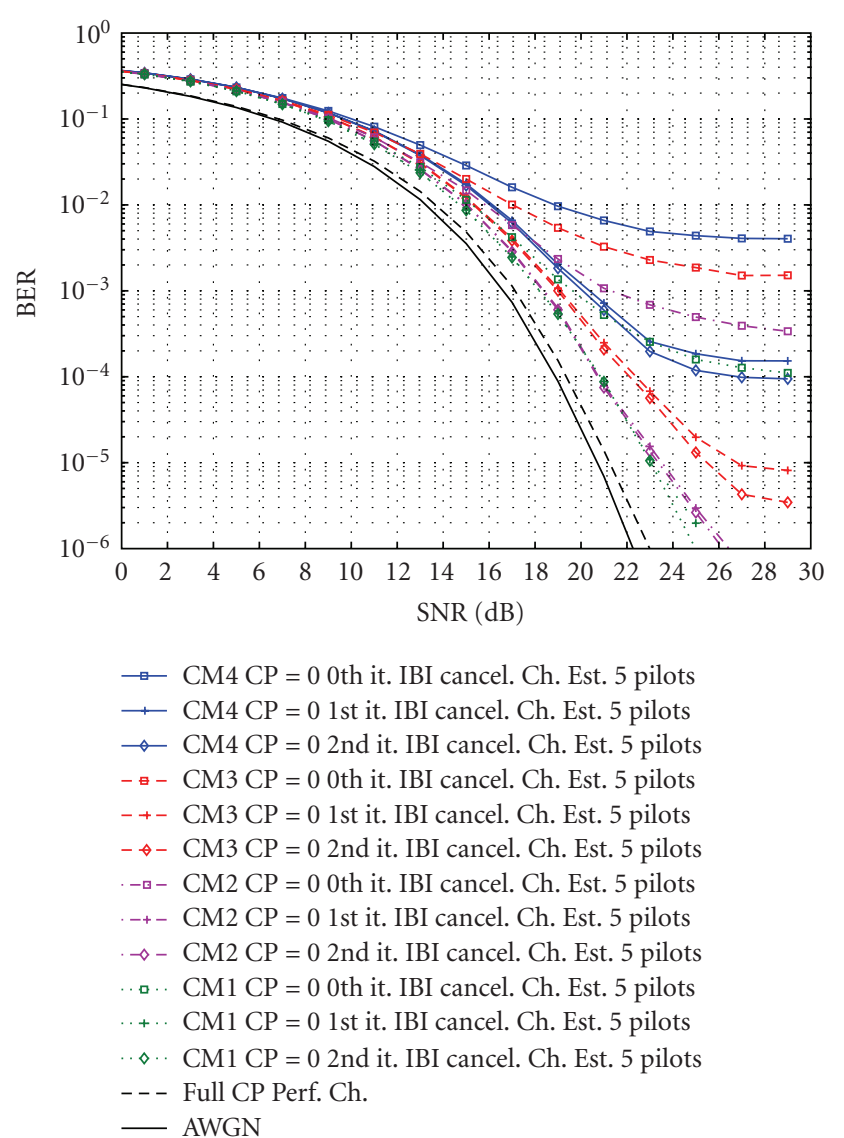

FIGURE 3: Receiver performance over different channel models, for $\mathrm{CP}=0$ and channel estimation with 5 pilot blocks.

when the channel estimation is performed with the IBI cancellation, the reduction in IBI for CP of 0,10 , and 20 symbol is more than that in the case of CP of length 50 . Moreover, using $50 \mathrm{CP}$ symbols with IBI cancellation, the estimator does not perform significantly closer to the full CP performance compared to shorter CP. This shows that using long $\mathrm{CP}$ is not necessary as it decreases the spectral efficiency without bringing significant performance improvements. We note that the IBI computation equation (19) can be evaluated directly. Alternatively the IBI terms can be multiplied with a weighting factor $\varepsilon_{n}(k)$ defined as

$$
\varepsilon_{n}(k)=\frac{\sum_{r=L_{p}+k}^{L_{c}-1} \hat{h}^{2}(r)}{\sum_{i=0}^{L_{c}-1} \hat{h}^{2}(i)},
$$

so as to reduce the impact of the interference power on the received signal during the IBI cancellation operation. Because of a slight performance improvement it provides over the direct case, we have employed the weighting factor in the IBI cancellation operations in all the channel estimation and equalization simulations.

The bit error rate (BER) performances of the iterative equalizer with soft IBI cancellation over all the UWB channel models are shown in Figures 3, 4, 5, and 6 for the (Pilot $=5$ symbols, $\mathrm{CP}=0)$, $(\mathrm{Pilot}=5$ symbols, $\mathrm{CP}=20)$, and $($ Pilot $=10$ symbols, $\mathrm{CP}=0),($ Pilot $=10$ symbols, $\mathrm{CP}=20)$ 


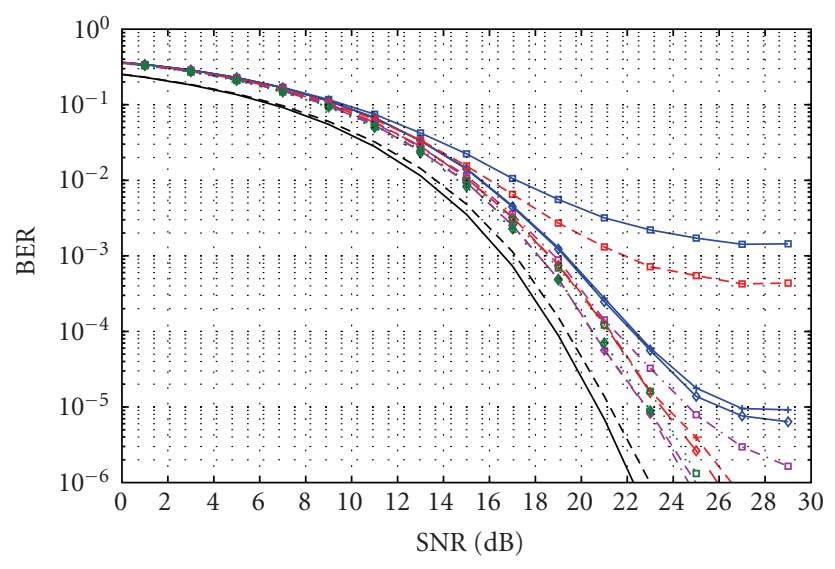

$\rightarrow \mathrm{CM} 4 \mathrm{CP}=20$ 0th it. IBI cancel. Ch. Est. 5 pilots

$\longrightarrow \mathrm{CM} 4 \mathrm{CP}=20$ 1st it. IBI cancel. Ch. Est. 5 pilots

$\rightarrow$ CM4 CP $=20$ 2nd it. IBI cancel. Ch. Est. 5 pilots

$-\because-\mathrm{CM} 3 \mathrm{CP}=20$ 0th it. IBI cancel. Ch. Est. 5 pilots

$-+-\mathrm{CM} 3 \mathrm{CP}=20$ 1st it. IBI cancel. Ch. Est. 5 pilots

$-\diamond-$ CM3 CP $=20$ 2nd it. IBI cancel. Ch. Est. 5 pilots

- $-\mathrm{CM} 2 \mathrm{CP}=20$ 0th it. IBI cancel. Ch. Est. 5 pilots

$-+-\mathrm{CM} 2 \mathrm{CP}=20$ 1st it. IBI cancel. Ch. Est. 5 pilots

$\diamond-\mathrm{CM} 2 \mathrm{CP}=20$ 2nd it. IBI cancel. Ch. Est. 5 pilots

-.. CM1 CP $=20$ 0th it. IBI cancel. Ch. Est. 5 pilots

$+\cdots \mathrm{CM} 1 \mathrm{CP}=20$ 1st it. IBI cancel. Ch. Est. 5 pilots

$\diamond \cdots \mathrm{CM} 1 \mathrm{CP}=20$ 2nd it. IBI cancel. Ch. Est. 5 pilots

- - Full CP Perf. Ch.

- AWGN

FIGURE 4: Receiver performance over different channel models, for $\mathrm{CP}=20$ and channel estimation with 5 pilot blocks.

scenarios, respectively. In each plot, simulations are presented for all CM1-CM4 UWB channel models so as comparisons are possible for the performance of the proposed receiver for different channels. In addition, the AWGN or the matched filter bound and the BER performance of the proposed receiver for the CM1 channel with full CP (no IBI) and perfect channel impulse response are also included in each plot as benchmarks. The full $\mathrm{CP}$ with perfect channel estimation curves for CM2-CM4 channels is not included for keeping the simplicity of the presentation. In all the plots, only the SNR computed over the data bits are considered instead of scaling the SNR over the data bits and the cyclic prefix in order to make the comparisons simpler. Notice in the figures that the use of no CP or short CP causes channel estimation errors. Naturally, using more pilot blocks lowers this error floor because the channel estimator improves not only its decisions but also the IBI cancellation performance with each additional pilot block. Notice that the use of a single pilot symbol does not provide a sufficiently good channel estimate and thus yields a performance degradation. However, when a moderate number of pilot symbols are employed, the iterative FD-MMSE equalizer with soft IBI canceller lowers the error floor significantly and even when no $\mathrm{CP}$ is employed it achieves a BER performance that is within $2 \mathrm{~dB}$ of that of the full CP case. Notice that in both $\mathrm{CM} 1$ and $\mathrm{CM} 2$ channels, 10 pilot symbols and $\mathrm{CP}$ lengths of 20 symbols in the proposed receiver scheme is

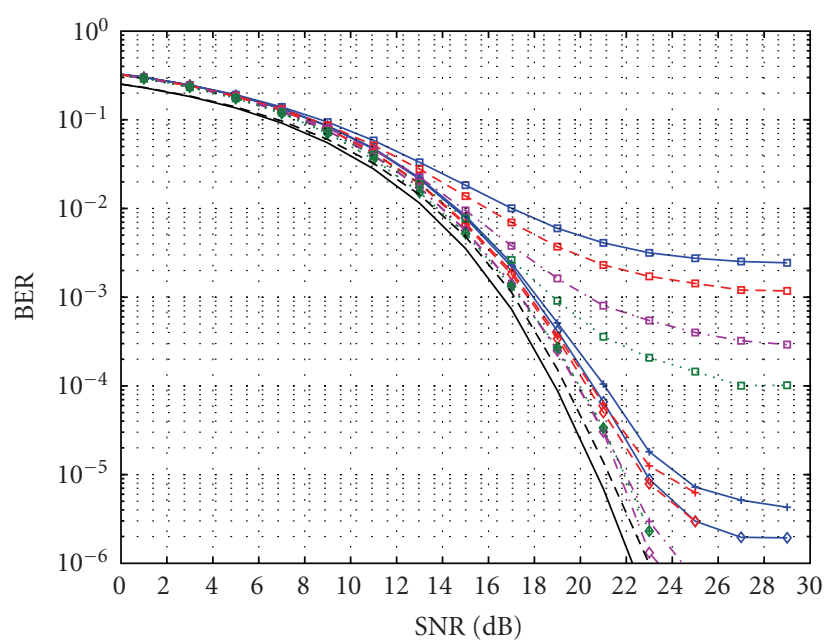

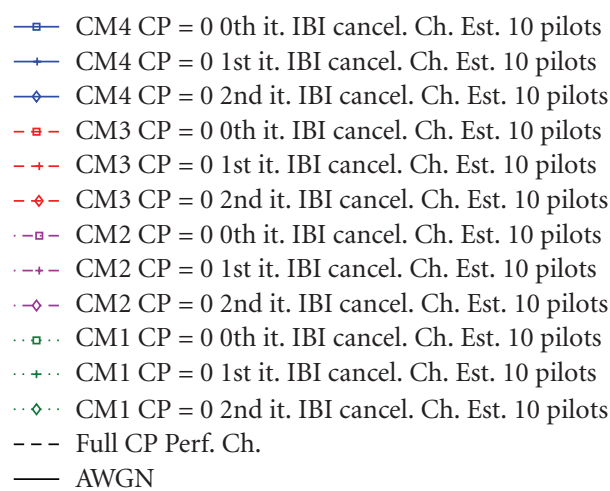

FIGURE 5: Receiver performance over different channel models, for $\mathrm{CP}=0$ and channel estimation with 10 pilot blocks.

enough to achieve performances sufficiently close to that of the AWGN or full CP bounds after only 2 iterations. As mentioned above, the weighting factor in (31) is used in all IBI computations in the equalization stage as well.

\section{Conclusion}

An iterative FD receiver is presented to combat with the deteriorating effects of using short CP for IR UB systems. An IBI estimation and cancellation scheme that can be used both with an FD channel estimator and with an FD MMSE equalizer is proposed. The FD channel estimator equipped with the IBI cancellation improves the channel estimates significantly. Employing iterative IBI cancellation within the back-end equalizer also improves the signal detection performance. We show with simulations that with moderate number of pilot blocks, the proposed receiver attains performances close to the full CP or AWGN bounds even in the case of no CP. Future works may include the analysis of the proposed system under parametric uncertainties such as the synchronization errors, channel estimation errors..., and as well as the derivation of analytical performance bounds for the channel estimation and equalization with IBI cancellation. 


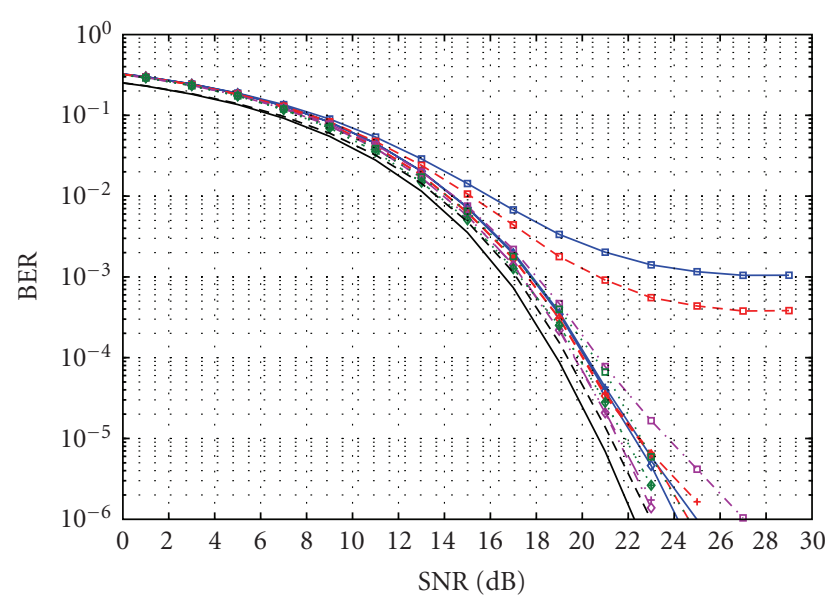

$\rightarrow \mathrm{CM} 4 \mathrm{CP}=20$ 0th it. IBI cancel. Ch. Est. 10 pilots $\rightarrow \mathrm{CM} 4 \mathrm{CP}=20$ 1st it. IBI cancel. Ch. Est. 10 pilots $\rightarrow \mathrm{CM} 4 \mathrm{CP}=20$ 2nd it. IBI cancel. Ch. Est. 10 pilots $-{ }_{-} \mathrm{CM} 3 \mathrm{CP}=20$ 0th it. IBI cancel. Ch. Est. 10 pilots $-+-\mathrm{CM} 3 \mathrm{CP}=20$ 1st it. IBI cancel. Ch. Est. 10 pilots $-\rightarrow-\mathrm{CM} 3 \mathrm{CP}=20$ 2nd it. IBI cancel. Ch. Est. 10 pilots $-\square-\mathrm{CM} 2 \mathrm{CP}=20$ 0th it. IBI cancel. Ch. Est. 10 pilots $-+-\mathrm{CM} 2 \mathrm{CP}=201$ st it. IBI cancel. Ch. Est. 10 pilots

$\leadsto-\mathrm{CM} 2 \mathrm{CP}=20$ 2nd it. IBI cancel. Ch. Est. 10 pilots

. ‥ CM1 CP $=20$ 0th it. IBI cancel. Ch. Est. 10 pilots

.. +. CM1 CP $=20$ 1st it. IBI cancel. Ch. Est. 10 pilots

• .. $\mathrm{CM} 1 \mathrm{CP}=20$ 2nd it. IBI cancel. Ch. Est. 10 pilots

- - Full CP Perf. Ch.

- AWGN

FIGURE 6: Receiver performance over different channel models, for $\mathrm{CP}=20$ and channel estimation with 10 pilot blocks.

\section{Appendix}

The derivation of (8) is as follows. We assume that each transmitted data block is composed of $N$ chips and it is equal or greater than the CIR $N \geq L_{c}$. Thus, when CP length is shorter than the CIR or even in the absence of CP, the IBI error in the $n$th data block are caused only from $(n-1)$ th data block. Defining the term $L_{d}=L_{c}-L_{p}$ as the difference between the CIR and CP, we can write the first element of the $n$th block that contains IBI error $r_{n}^{\mathrm{IBI}}(0)$ by convolving the CIR and transmitted data block:

$$
\begin{aligned}
r_{n}^{\mathrm{IBI}}(0)= & d_{n}(0) h(0)+\sum_{r=0}^{L_{p}-1} h\left(L_{p}-r\right) d_{n}\left(N-L_{p}+r\right) \\
& +\sum_{r=L_{p}+1}^{L_{c}-1} h(r) d_{n-1}\left(N+L_{p}-r\right) .
\end{aligned}
$$

If the CP length were sufficient, then the first term of the $n$th received block $r_{n}(0)$ would be

$$
\begin{aligned}
r_{n}(0)= & d_{n}(0) h(0)+\sum_{r=0}^{L_{p}-1} h\left(L_{p}-r\right) d_{n}\left(N-L_{p}+r\right) \\
& +\sum_{r=L_{p}+1}^{L_{c}-1} h(r) d_{n}(N-r) .
\end{aligned}
$$

Then, the IBI error in the first element of the received vector $e_{n}(0)$ is expressed as

$$
\begin{aligned}
e_{n}(0) & =r_{n}^{\mathrm{IBI}}(0)-r_{n}(0) \\
& =\sum_{r=L_{p}}^{L_{c}-1} h(r)\left[d_{n-1}\left(N+L_{p}-1-r\right)-d_{n}(N-r)\right] .
\end{aligned}
$$

Similarly, the IBI error terms $e_{n}(k)$ for $k=1, \ldots, L_{d}-2$ are written as

$$
\begin{aligned}
& e_{n}(1) \\
& \quad=\sum_{r=L_{p}+2}^{L_{c}-1} h(r)\left[d_{n-1}\left(N+L_{p}+1-r\right)-d_{n}(N-r+1)\right], \\
& e_{n}(2){ }_{r=L_{p}+3} h(r)\left[d_{n-1}\left(N+L_{p}+2-r\right)-d_{n}(N-r+2)\right], \\
& \quad \vdots \quad L_{c}-1 \text {. } \\
& \quad=h\left(L_{c}-1\right)\left[d_{n-1}(N-1)-d_{n}\left(N-L_{c}+L_{d}\right)\right] .
\end{aligned}
$$

As a result, we can obtain the closed form expression of the IBI error as

$e_{n}(k)$

$$
\begin{array}{r}
=\sum_{r=L_{p}+k+1}^{L_{c}-1} h(r)\left[d_{n-1}\left(N+L_{p}+k-r\right)-d_{n}(N-r+k)\right], \\
k=0,1, \ldots, L_{d}-2 .
\end{array}
$$

Notice that by definition $e_{n}(k)=0$ for $k=L_{d}-1, \ldots, N-1$.

\section{Acknowledgment}

This work is supported by The Scientific and Technological Research Council of Turkey (TÜBİTAK) EEEAG under grant no. 105E077 and by The Boğaziçi University Research Projects Fund under grant no. 5181.

\section{References}

[1] H. Sari, G. Karam, and I. Jeanclaude, "Transmission techniques for digital terrestrial TV broadcasting," IEEE Communications Magazine, vol. 33, no. 2, pp. 100-109, 1995.

[2] D. Falconer, S. L. Ariyavisitakul, A. Benyamin-Seeyar, and B. Eidson, "Frequency domain equalization for single-carrier broadband wireless systems," IEEE Communications Magazine, vol. 40, no. 4 , pp. 58-66, 2002. 
[3] F. Pancaldi, G. M. Vitetta, R. Kalbasi, N. Al-Dhahir, M. Uysal, and H. Mheidat, "Single-carrier frequency domain equalization: a focus on wireless applications," IEEE Signal Processing Magazine, vol. 25, no. 5, pp. 37-56, 2008.

[4] J. R. Foerster, "Channel modeling sub-committee repot (final),” Tech. Rep. P802.15-02/368r5-SG3a, IEEE P802.15 Working Group for Wireless Personal Area Networks (WPANs), 2002.

[5] Y. Ishiyama and T. Ohtsuki, "Performance evaluation of UWBIR and DS-UWB with MMSE-Frequency Domain Equalization (FDE)," in Proceedings of the IEEE Global Telecommunications Conference (GLOBECOM '04), vol. 5, pp. 3093-3097, December 2004.

[6] Y. Wang, X. Dong, P. H. Wittke, and S. Mo, "Cyclic prefixed single carrier transmission in ultra-wideband communications," IEEE Transactions on Wireless Communications, vol. 5, no. 8, pp. 2017-2021, 2006.

[7] S. Morosi and T. Blanchi, "Frequency domain detectors for ultra-wideband indoor communications," IEEE Transactions on Wireless Communications, vol. 5, no. 10, pp. 2654-2658, 2006.

[8] S. Morosi and T. Bianchi, "Frequency domain detectors for ultra-wideband communications in short-range systems," IEEE Transactions on Communications, vol. 56, no. 2, pp. 245 $253,2008$.

[9] K. Kobayashi, T. Ohtsuki, and T. Kaneko, "Performance evaluation of ultra wideband-impulse radio (UWB-IR) with iterative equalization based on energy spreading transform (EST)," in Proceedings of the IEEE Vehicular Technology Conference (VTC '05), vol. 2, pp. 996-1000, September 2005.

[10] P. Kaligineedi and V. K. Bhargava, "Frequency-domain turbo equalization and multiuser detection for DS-UWB systems," IEEE Transactions on Wireless Communications, vol. 7, no. 9, pp. 3280-3284, 2008.

[11] Y. Wang and X. Dong, "Frequency-domain channel estimation for SC-FDE in UWB communications," IEEE Transactions on Communications, vol. 54, no. 12, pp. 2155-2163, 2006.

[12] H. Sato and T. Ohtsuki, "Frequency domain channel estimation and equalisation for direct sequence-ultra wideband (DS-UWB) system," IEE Proceedings, vol. 153, no. 1, pp. 9398, 2006.

[13] P. J. W. Melsa, R. C. Younce, and C. E. Rohrs, "Impulse response shortening for discrete multitone transceivers," IEEE Transactions on Communications, vol. 44, no. 12, pp. 1662$1672,1996$.

[14] T. Hwang and Y. Li, "Iterative cyclic prefix reconstruction for coded single-carrier systems with frequency-domain equalization(SC-FDE)," in Proceedings of the 57th IEEE Semiannual Vehicular Technology Conference (VTC '03), vol. 3, pp. 1841-1845, April 2003.

[15] A. Gusmão, P. Torres, R. Dinis, and N. Esteves, "A reduced-CP approach to SC/FDE block transmission for broadband wireless communications," IEEE Transactions on Communications, vol. 55, no. 4, pp. 801-809, 2007.

[16] A. Gusmão, P. Torres, R. Dinis, and N. Esteves, "A turbo FDE technique for reduced-CP SC-based block transmission systems," IEEE Transactions on Communications, vol. 55, no. 1, pp. 16-20, 2007.

[17] H. Liu and P. Schniter, "Iterative frequency-domain channel estimation and equalization for single-carrier transmissions without cyclic-prefix," IEEE Transactions on Wireless Communications, vol. 7, no. 10, pp. 3686-3691, 2008.

[18] S. Yoshida and T. Ohtsuki, "Improvement of bandwidth efficiency of UWB-IR and DS-UWB with frequency-domain equalization (FDE) based on cyclic prefix reconstruction," in Proceedings of the IEEE Vehicular Technology Conference (VTC '05), vol. 2, pp. 25-28, September 2005.

[19] S. Yoshida and T. Ohtsuki, "Effect of imperfect channel estimation on the performance of UWB-IR with FrequencyDomain Equalization (FDE) and Cyclic Prefix (CP) reconstruction," in Proceedings of the IEEE Pacific Rim Conference on Communications, Computers and Signal Processing (PACRIM '07), pp. 342-345, Victoria, BC, Canada, August 2007.

[20] Y. Wang and X. Dong, "A time-division multiple-access SCFDE system with IBI suppression for UWB communications," IEEE Journal on Selected Areas in Communications, vol. 24, no. 4, pp. 920-926, 2006.

[21] C.-J. Park and G.-H. Im, "Efficient cyclic prefix reconstruction for coded OFDM systems," IEEE Communications Letters, vol. 8, no. 5, pp. 274-276, 2004.

[22] W. Zhuang, X. Shen, and Q. Bi, "Ultra-wideband wireless communications," Wireless Communications and Mobile Computing, vol. 3, no. 6, pp. 663-685, 2003.

[23] M. Morelli, L. Sanguinetti, and U. Mengali, "Channel estimation for adaptive frequency-domain equalization," IEEE Transactions on Wireless Communications, vol. 4, no. 5, pp. 2508-2518, 2005.

[24] S. Papaharalabos, P. Sweeney, B. G. Evans et al., "Modified sum-product algorithms for decoding low-density paritycheck codes," IET Communications, vol. 1, no. 3, pp. 294-300, 2007.

[25] M. Tuchler and J. Hagenauer, "Turbo equalization using frequency domain equalizers," in Proceedings of the Allerton Conference, Monticello, Ill, USA, October 2000.

[26] K. Li, X. Wang, G. Yue, and L. Ping, "A low-rate codespread and chip-interleaved time-hopping UWB system," IEEE Journal on Selected Areas in Communications, vol. 24, no. 4, pp. 864-870, 2006. 